\title{
Investigate the Relationship Between Sleep Quality and Some Blood Parameters in Thalassemia Major Patients Referred to Cooley's ward at Motahari Hospital in Jahrom, Iran
}

\author{
Zohreh Badiyepeymaiejahromi ${ }^{1}$, Farzad Poorgholami², \\ Mahboobeh Taghizadeganzadeh ${ }^{2}$, Zahra Pishgar ${ }^{2}$ and Nehleh Parandavar ${ }^{1 *}$ \\ ${ }^{1}$ Research Centre for social Determinants of Health, \\ Jahrom University of Medical Sciences, Jahrom, Iran. \\ ${ }^{2}$ Department of Nursing, Jahrom University of Medical Sciences, Jahrom, Iran.
}

http://dx.doi.org/10.13005/bbra/2158

(Received: 13 April 2016; accepted: 01 June 2016)

\begin{abstract}
Sleep is considered as one of the basic physiological needs of the human body, but the discomfort of physical and mental illness can interfere with a person's sleep. Thalassemia major, a disease characterized by reduced blood hemoglobin defect or nonproduction of one of the branches leads to disruption in the process of hematopoiesis and as a result is anemia. The aim of this study was to evaluate sleep quality in patients with thalassemia major referred to Motahari Hospital and its relationship with some blood parameters. This cross-sectional study was conducted in 2014 . 60 patients with definitive diagnosis of thalassemia who dependent blood transfusion and for transfusion and clinical examination referred to Cooley's ward in the Motahari hospital in Jahrom participated. The subjects responded to questions Pittsburgh Sleep Quality Index and demographic data, information on indicators of blood and medications were taken from their cases. Based on statistical analysis, Mean score of patients' quality sleep was $4.43 \pm 2.87$, and 41 cases of the patients $(68.33 \%)$ had good sleep quality and 19cases $\mathbf{( 3 1 . 6 6 \% )}$ had poor sleep quality. Among the demographic variables, age was positively correlated with sleep quality, but gender had no significant relationship with sleep quality. Also there was no significant relationship between sleep quality with the mean doses of medications to control blood iron, hemoglobin, ferritin, level of liver enzymes and the frequency of blood transfusion in the last six months. The results of this study show the need to pay more attention to sleep quality of patients with thalassemia. It seems that therapeutic factors such as medications and serum levels of some blood parameters such as hemoglobin, ferritin and ... was not associated with sleep quality and the importance of personality, psychological and environmental factors in this field to be working.
\end{abstract}

Key words: Thalassemia, anemia, sleep quality, patients.

Thalassemia major is a chronic genetic blood disease which results from deficiency in synthesis of one or several polypeptide chains of globin and is considered as a single-gene disorder that transmits by a recessive autosomal pattern ${ }^{1}$. Its prominent sign is deficiency or absence of one

\footnotetext{
* To whom all correspondence should be addressed. Tel.: 09177916704;

E-mail: shaghayegh_ne.2001@yahoo.com
}

of blood hemoglobin units which leads to disorder in hematopoiesis and the resultant severe anemia². Thalassemia has a considerable prevalence in some regions of Iran including Isfahan, Fars, and Bushehr ${ }^{3}$. Because of high number of affected people and wide range of physical disorders resulting from this disease, it is known as one of the most important chronic physical disorders in Iran that makes several physical, psychological and social problems for the patients ${ }^{4}$. Keeping the 
hemoglobin of these patients high through repeated blood infusion by the recommended rate $(10 \mathrm{~g} / \mathrm{dL})$ assures their survival and growth, but the excess iron accumulates in the body which ends up in the most significant problem of thalassemia patients being iron overload and hemosiderosis. Finally, cardiac disease and disorders in glands functions results in their death when they are young ${ }^{2}$. On the other hand, extramedullary hematopoiesis in Thalassemia major causes bone malformations in head and neck like tight occlusion of the teeth, delay in formation of paranasal sinuses and other similar disorders. Additionally, there are remarkable systemic problems which may affect head and neck ${ }^{5}$ and cause sleep disturbances.

Sleep is one of the vital needs of human that plays an important role in recovering the lost strength and relief from fatigue resulting from daily life activities and is known as an important criterion in protecting human physical and psychological health ${ }^{6}$. Any kind of disorder in its natural procedure could reduce people efficiency in addition to causing psychological problems ${ }^{7}$. Sleep removes fatigue and prepares human again for daily activities through different mechanisms ${ }^{8}$. Since complaints about sleep are common and poor quality sleep can be indicator of many diseases, paying enough attention to sleep quality is of importance ${ }^{9}$. Sleep induced problems can lead to reduction in appetite, physical and mental fatigues, lack of concentration, nervous and physiological changes, decrease in the speed of information analysis, increase in human errors and accidents, loss of consciousness, depression and anxiety ${ }^{10-}$ ${ }^{12}$. Besides, increase in cardiovascular diseases, digestive system disorders and tendency for using drugs may happen following sleep disturbances ${ }^{13}$.

Damage to personal function, mental fatigue, memory disorder, comprehension changes and judgment ability has also been mentioned ${ }^{14}$. On the other hand, physical and psychological diseases can interfere with sleep ${ }^{15}$ and lots of physical diseases disturb sleep and disrupt consciousness ${ }^{16}$. Almost all physical diseases that are accompanied by pain or considerable discomfort, or those which are caused by metabolic disorders could affect both quality and quantity of sleep negatively ${ }^{17}$.

Several epidemiologic investigations about the relationship of the quantity and quality of night sleep with prevalence and incidence of some chronic diseases have been performed during recent years. Increase or decrease in the duration of night sleep has had relationship with increase in prevalence and incidence of diabetes, and inappropriate control of blood sugar ${ }^{18}$. Diabetes prevalence and disorder in glucose tolerance augments with sleep length of less than 6 hours or more than 9 hours, and diabetic people have less sleep time compared to normal sleep in the society ${ }^{19}$. Partial or complete night sleep deprivation leads to increase in sympathetic system activity, increase in night level of blood cortisol, and increase in growth hormone level during the day $^{20}$.

Considering numerous problems resulting from beta-thalassemia disease and its treatment methods, it is expected that these people suffer from sleep disorders too, and as it was mentioned earlier sleep disorder affects these people physiologic parameters. Treatment personnel as people who are actively participating in all prevention, treatment and recovery steps of the thalassemia disease need to be aware of negative physiologic and psychologic effects of this disease, so that they can design and execute care programs for ameliorating patients' health level. The aim of this study is to determine the relationship between sleep quality and some blood parameters in thalassemia patients major referring to Cooley's ward at Motahari hospital of Jahrom.

\section{METHODS}

This cross sectional study was performed in 2014 through convenience sampling on 60 patients with definitive thalassemia major diagnosis who were dependent on blood infusion and used to refer to Cooley's department of Motahari hospital for monthly blood infusion and clinical examinations.

For data collection, questionnaires were used. The researcher-made demographic questionnaire included age, gender, education, marital status and splenectomy. Furthermore, quality of sleep was measured using the PSQI. The Pittsburg Sleep Quality Index (PSQI) is composed of 10 questions to measure the sleep quality of the adult during the month that preceded the interview. 
This is divided into seven areas: subjective sleep quality (C1), sleep latency (C2), sleep duration (C3), habitual sleep efficiency (C4), sleep disturbances (C5), and use of sleep medication (C6) and daytime dysfunction (C7). The client self-rates each of these seven areas of sleep. For each area score is attributed, varying from 0 (zero) to 3 (three) scale, whereby 3 reflects the negative extreme on the Likert Scale. The final score is obtained from the sum of the scores from the seven areas, varying from 0 to 21, being the better the quality of sleep with the lower score obtained. A global sum of" 5 "or greater indicates "poor"sleeper. Buysse et al. reported a global PSQI score $>5$ had a sensitivity of $89.6 \%$ and specificity of $86.5 \%$ (kappa $=0.75$, $p$ $<.001$ ) in distinguishing subjects with good sleep quality from those with poor sleep ${ }^{21}$. This questionnaire has been translated and validated for Iranian population ${ }^{22}$.

Organized survey was utilized to collect data from patients, and cooley's department personnel read the statements of questionnaire for the patients who had the needed criteria to enter study and were volunteer for participating in the research, and their answers were recorded. Other data regarding the medical information were then obtained from their files.

The research design was approved by research board and ethics committee and introduction letter was presented by research assistant to the manager of Motahari hospital, and testimonial was received from all participating patients and they were assured that all the data will be kept secret.

After collection, the data were entered in SPSS V.16 software and were analyzed by descriptive tests (frequency, mean, and standard deviation) and analytical tests (Spearman's correlation coefficient).

\section{RESULTS}

60 thalassemia major patients with average age of $24.07 \pm 8.72$ and average times blood infusion in the last 6 months $8 \pm 2.5$ participated in this study. The participating patients consisted of 26 (43.3\%) males and 34 (53.7\%) females, 49 singles (81.7\%) and 11 married (46.7\%), 28 (43.6\%) people with under diploma education and 23 (38.3\%) people with diploma, and 9 people (15\%) with education higher than diploma. 33 people (55\%) of them had been splenectomized.

Table 1. Relationship between sleep quality of patients and the mean of hemoglobin, ferritin, liver enzymes and number of blood transfusion

\begin{tabular}{|c|c|c|c|c|}
\hline \multirow{2}{*}{$\begin{array}{l}\text { Sleep quality } \\
\text { Mean of blood parameters }\end{array}$} & \multicolumn{2}{|c|}{ Quality of sleep } & \multirow[t]{2}{*}{ P-value } & \multirow[t]{2}{*}{$\mathrm{r}$} \\
\hline & Good sleep quality & Poor sleep quality & & \\
\hline Hemoglobin(M士SD) & $9.99 \pm 0.53$ & $9.92 \pm .055$ & 0.44 & 0.12 \\
\hline 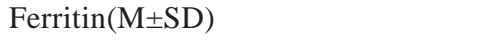 & $2196.40 \pm 1499.33$ & $2237.50 \pm 1708.57$ & 0.94 & 0.01 \\
\hline SGOT(M \pm SD) & $27.59 \pm 18.20$ & $49.16 \pm 43.39$ & 0.28 & 0.17 \\
\hline SGPT(M $\pm S D)$ & $36.70 \pm 30.62$ & $68.25 \pm 77.68$ & 0.3 & 0.17 \\
\hline Number of blood transfusion(M $\pm \mathrm{SD})$ & $8.18 \pm 2.76$ & $7.54 \pm 1.75$ & 0.2 & 0.21 \\
\hline
\end{tabular}

Table 2. Relationship between sleep quality and mean dose of blood iron reducer drugs

\begin{tabular}{|c|c|c|c|c|}
\hline \multirow{2}{*}{$\begin{array}{l}\text { Sleep quality } \\
\text { Mean of blood iron reducer drugs }\end{array}$} & \multicolumn{2}{|c|}{ Quality of sleep } & \multirow[t]{2}{*}{ P-value } & \multirow[t]{2}{*}{$\mathrm{r}$} \\
\hline & Good sleep quality & Poor sleep quality & & \\
\hline 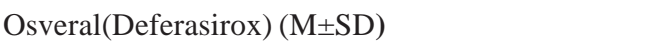 & $1.40 \pm 1.75$ & $2.25 \pm 1.86$ & 0.18 & 0.22 \\
\hline 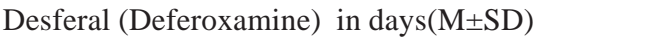 & $2.50 \pm 2.24$ & $2.16 \pm 2.32$ & 0.7 & 0.06 \\
\hline 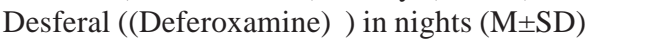 & $3.19 \pm 3.12$ & $2.41 \pm 2.99$ & 0.54 & 0.1 \\
\hline 1,2-dimethyl-3-hydroxypyrid-4-one(L1) (M $\pm \mathrm{SD})$ & $1.15 \pm 2.57$ & $0.91 \pm 1.97$ & 0.82 & 0.03 \\
\hline $\begin{array}{ll}\text { Using Hydroxyurea } & \text { Yes }(\%) \\
& \end{array}$ & $\begin{array}{c}1(3.7) \\
26(96.3)\end{array}$ & $\begin{array}{c}1(8.3) \\
11(917)\end{array}$ & 0.55 & 0.09 \\
\hline
\end{tabular}


According to the statistical analyses, the mean score for patients' sleep quality was $4.43 \pm 2.87$ and 41 people (68.33\%) had good sleep quality and 19 people (31.66\%) had poor sleep quality. On average patients went to bed around 20.98 \pm 5.99 , but it took them $1.05 \pm 0.91$ hours to sleep.

Spearman's test showed that there were no statistically significant correlations $(p>0.05)$ between sleep quality and the mean of hemoglobin, ferritin, liver enzymes, and the number of blood transfusion during the last six months (Table 1).

Regarding the mean age, a statistically significant difference $(\mathrm{p}=0.03, \mathrm{r}=0.34)$ was seen between the group of good sleep quality (22.07 \pm 7.08$)$ and poor group (28.33 \pm 1.71 ). But there was no statistically significant relationship between sex and sleep quality ( $\mathrm{p}=0.62, \mathrm{r}=0.08$ ).

Based on table 2 findings, Spearman's test demonstrates that there is not any significant difference ( $>0.05$ ) between the average dose of blood iron reducer drugs (Osveral, Desferal, 1,2dimethyl-3-hydroxypyrid-4-one(L1), Hydroxyurea) and thalassemia major patients' sleep quality.

\section{DISCUSSION}

Nowadays, thalassemia major as the most common hereditary disorder in the world is not considered as a fatal disease. Present health cares and treatments and special attention to their life quality have caused amelioration of these people lives level. Current study was conducted with the aim of studying the presence of sleep disorder in this group of patients and its relationship with some blood parameters. Like the study of Azarkeivan et al., the mean age of patients shows that affected people are young (23). The results of current study are indicative of sleep disorder presence in less than one third of studied people, so that the distribution of sleep disorder in study population has been $30.8 \%$ (24), but this number is much less than the normal and healthy available under study population (61\% unfavorable sleep quality) (25). The performed studies on different chronic diseases like the study of Aslani et al. and Parker et al.'s study on patients with cardiac failure (26, 27), and the researches of Mei-Fen Pai et al., (28) and Baraz et al., (29) on hemodialysis patients show the high prevalence of sleep disorders which is not consistent with the present study. Difference in the number of people in this study could be the reason for this inconsistency. Number of people with sleep disorder has been obtained less than normal population because of the low number of participants in this study. On the other hand, there were no studies available which had evaluated the sleep disorder in this population of patients.

Besides, the results show that sleep quality in people with higher age average is more unfavorable that is similar to the results of Emkani et al. who observed a significant relationship between age and sleep quality ${ }^{25}$.

Sleep disorder in patients with chronic pain could increase sensitivity to pain, augment attention to pain, interfere with daily function, and produce a permanent cycle of sleep disturbance, increasing pain and even depression. Although few patients had reported depression as a disturbing factor for sleep in the research of Afkham ebrahimi et al. ${ }^{30}$, presence of apparatus for Desferal injection and carrying it and sometimes its existence during sleep might be a reason for sleep disturbance and taking long to start sleep.

In current study, no statistical difference was observed between two groups for ferritin, but its overall mean in two groups was $2209.05 \pm 1543.86$ which is in normal range expected in thalassemia patients. In the study of Azarkeivan et al., ferritin level was 1913.8ng/dl and Lakzai reported it as 1000$2000^{23,31}$. The best serum ferritin level in major thalassemia patients is less than $1000-1500 \mu \mathrm{g} / \mathrm{L}$. serum ferritins higher than 2500 increases risk of cardiac disease and death. Maximum physiologic limit is 4000, higher than which is related to cellular damage $^{32,33}$. The results indicate lack of relationship between sleep disturbance and blood levels of ferritin. Furthermore, since ferritin levels in this study are in normal interval and are acceptable, it is concluded that iron reduction treatment had been performed efficiently. It should also be noted that although ferritin level is normal in major thalassemia patients, this phenomenon could lead into growth delay in them ${ }^{34}$. In the study conducted by Talaeepoor, the aim of which was to compare the serum ferritin level between major thalassemia patients in the group being treated by Desferal alone and the group being treated by Desferal and Osveral in Bandarabbas city, no significant difference was seen in ferritin level reduction between the two groups. A mild leukocytosis and 
increase in liver enzymes was demonstrated in this study, but they were not significant ${ }^{35}$.

Additionally, the study results showed that the two favorable and unfavorable sleep quality groups did not have significant difference for hemoglobin level, but the mean of hemoglobin level in people with unfavorable sleep quality is a little lower. The hemoglobin level in these patients is similar to that obtained in the research of Azarkeivan et al. which is in the expected interval for thalassemia patients ${ }^{23}$.

Azarkeivan et al. also reported SGOT level (44.48 \pm 23.1$)$ and SGPT level(48.2 \pm 35.37$)$ (23). SGOT level in current study is lower than that reported by Azarkeivan, but reported levels for SGPT are similar to those of present study. No statistically significant difference was observed between the two groups. Talaeepoor et al. demonstrated that using Desferal and Osveral resulted in a subtle leukocytosis and increase in liver enzymes ${ }^{35}$.

Increased liver enzymes in these patients are indicative of liver damage which could be due to several reasons including: viral hepatitis infections which were not examined in this study, extramedullary hematopoiesis which might have happened as the result of lack of bone marrow inhibition and liver overacting.

It was seen in current study that the mean of blood infusion times did not affect sleep quality in the two groups, so that sleep quality and the number of blood reception times did not differ significantly between the two groups. Furthermore, the sleep quality was not significantly different between two groups, despite the better sleep quality in people who had more Desferal reception times and higher L1 used.

According to numerous reports from different countries around the world, it seems that in today medicine world, the life of these patients has extended ${ }^{36}$. Disease identification in the primary stages, drugs available for patients, governmental and familial supports, and have all resulted in ameliorated physical and psychological attitude in this group of patients. Of course, psychological evaluation should be performed in different studies.

The findings of Azarkeivan et al. are indicative of decrease in facial malformations following better acceptance of disease or selfconception of the patient due to available treatment methods ${ }^{23}$. It also seems that stronger familial relationships, religious believes, and more cooperation in Iranian families has caused problems induced by the disease to be more acceptable for affected people.

Small sample volume was one of the limitations in this study. It is recommended that future studies get done with more patients and life quality and psychological evaluations get evaluated simultaneously.

\section{CONCLUSION}

Based on the findings of different studies, lots of physical diseases could cause sleep disturbance. Therefore, this problem should be considered in evaluation and treatment of different physical patients including thalassemia affected people. The results of current study highlight the necessity of paying more attention to sleep quality of thalassemia patients. It seems that treatment factors like drugs and serum levels of some blood indices including hemoglobin, ferritin, and etcetera do not correlate with sleep quality and it might be efficient to consider the personality and psychological factors.

\section{ACKNOWLEDGMENTS}

This paper presents the results of a research project approved by the Jahrom University of Medical Sciences, Ethics Committee code of JUMS.REC.1392.08. Hereby, the authors give their thanks to all patients who participated in the study, and appreciate the help of the ViceChancellor of Jahrom University of Medical Sciences who arranged for financial support of this project.

\section{REFERENCES}

1. Hosseini SH, Khani H, Khalilian A. R, Vahidshahi K. Psychological aspects in young adults with Beta- major,control group. Journal of Mazandaran University of Medical Sciences. 2007; 17(59): 51-60.

2. Zarezadeh Y, Ebrahimi E, Gheydari ME, Amani A, Jalili A. Effects of Aerobic Exercise on Body Iron Indices in healthy individuals and in patients with major thalassemia. Journal of Kurdestan Medical Sciences 2010; 5(18). 
3. Saki N, Dehghanifard A, Kaviani S, Jalalifar MA, Mosavi SH, Aleali Kh, Rahim F. Beta thalassemia: epidemiology and diagnostic and therapeutic strategies in Iran. Journal of Genetics in the Third Millennium 2012; 10(1): 2674-2683.

4. Yazdi Kh, Sanagu A, Jouybari L. Psychosocial, social and financial problems with coping strategies among families with thalassemic patients. Journal of Gorgan University of Medical Sciences. 2007; 9 (2):71-5.

5. Barjes N, Sonbolestan SM, Nemati Sh, Danesh Z, Mokhtarenezhad F, Abdyazdan Z. Manifestation of Ear, nose and throat of patients with thalassemia major. Journal of Iran Pediatrics 2007; 17(1): 15-18.

6. Araste M. Evaluation of insomnia in medical students of Kurdistan University. Sci J Kurdistan Univ Med Sci. 2007; 12(3):58-63.

7. Nobahar M, Vafaee A. Elderly sleep disturbances and management. Geriatric. 2007; 2(4): 263-8.

8. Barsam T. Sleep quality changes in workers exposed to extremely low frequency electromagnetic fields in high voltage electricity substations in Kerman district [dissertation]. Kerman: Kerman University; 2011.

9. IzadiAvnjy FS, Hajbaghery MA, Afazl MR. Quality of sleep and its related factors in the hospitalized elderly patients of Kashan hospitals in 2007. Fayz J. 2008; 12(4):52-9.

10. Mosavi F, Golestan B, Mohseni E, Tabatabai R. Sleep quality and related factors in interns and externs of Tehran Islamic Azad university medical students. Islamic Azad Univ Med Sci J. 2010; 20(4):4-9.

11. Salehi K, Alhani F, Sadegh-Niat K, Mahmoudifar Y, Rouhi N. Quality of sleep and related factors among Imam Khomeini hospital staff nurses. Iran Univ Med Sci J. 2010; 23(63):18-25.

12. Mokarami H, Kakvyy H, Dehdashti AR, Jahani Y, Ebrahimi H. Comparison of general health status and quality of sleep, shift workers in a workplace press. Kermanshah Univ Med Sci Res J. 2010; 14(3):237-43.

13. Saremi M, KhaniJzny R, Tassi P. Comparison of fatigue, sleep quality and quantity at once old and young farmers. Med Res. 2008; 32(2): 135-9.

14. Nojoomi M, Ghalebandi MF, Akhbari R, Gorji R. Sleep pattern and prevalence of sleep disturbances in medical students and specialist residents. Medical Science Journal of Islamic Azad University, tehran Medical Unite 2009;19(1): 55-59.

15. Alimarmae N. Factors affecting sleep from the perspective of patients admitted to the Besat medical center in Sanandaj in 1380. Scientific
Journal of Nursing and Midwifery faculty of Gorgan 2005; 2(2): 37-41.

16. Lamberg L. Insomnia shows strong link to psychiatric disorders. Psychiatric News 2005; 40: 21-3.

17. Fiorentino L, Ancoli-Israel S. Insomnia and its treatment in women with breast cancer. Sleep medicine Reviews 2006; 10: 419-29.

18. Nakajima H, Kaneita Y, Yokoyama E, Harano S, Tamaki T, Ibuka E, et al. Association between sleep duration and hemoglobin A1C level. Sleep Med 2008; 9: 745-52.

19. Gottlieb DJ, Punjabi NM, Newman AB, Resnick HE, Redline S, Baldwin CM, et al. Association of sleep time with diabetes mellitus and impaired glucose tolerance. Arch Internal Med 2005; 165: 863-7.

20. Spiegel K, Leproult R, Van Cauter E. Impact of sleep debt on metabolic and endocrine function. Lancet. 1999; 354: 1435-9.

21. Buysse DJ, Reynolds CF, Monk TH, Berman SR, Kupfer DJ. The Pittsburgh Sleep Quality Index: A new instrument for psychiatric practice and research. Psychiatry Res. 1989; 28: 193-213.

22. Kakooei H, Zare S, Akhlaghi A, Panahi D. Evaluation of sleep quality in bus drivers in Tehran. Traf Manag Stud. 2010; 5(16):1-10.

23. Azarkeivan A. Hajibeigy B, Afradi H, Eslami, M,Ghazizadeh Sh, Shabeh Pour Z. Evaluation of clinical conditions of thalassemic patients having referred to Adult Thalassemia Center, Tehran. Sci J Iran Blood Transfus Org 2011; 8(1): 32-41.

24. Ghoraishi SA, Aghajani AH. Sleep quality in Zanjan university medical students. Tehran Univ Med Sci J. 2008; 66(1):61-7.

25. Emkani M, Khanjani N. Sleep quality and its related factors in intercity bus drivers. Iranian Journal of Military Medicine 2012:14(2): 137141.

26. Aslani Y, Eetemadifar Sh, Aliakbari F, Heydari A. Sleep disorders in patients with heart failure hospitalized in Hajar hospital in Shahrekord in 1382. Journal of Shahrekord University of Medical Sciences 2007; 9(1): 44-49.

27. Parker KP,Dunbar SB.sleep and heart failure.J Cardiovasc Nurse.2002;17(1):30-41.

28. Mei-Fen Pai1, Shih-Ping Hsu1, Shao-Yu Yang1, Tai-I Ho1, Chun-Fu Lai1 and Yu-Sen Peng11Division of Nephrology, Department of Internal Medicine, Far Eastern Memorial Hospital, Taipei, Taiwan ,29(6): 673-677

29. Baraz Sh, Mohammadi E, Bromand B. Relationship between sleep quality, quality of life and some blood parameters in patients under treatment with hemodialysis. Journal of 
Shahrekord University of Medical Sciences 2007; 9(4): 67-74.

30. Afkham Ebrahimi A, Ghale Bandi M.F, Salehi M, Kafian Tafti AR, Vakili Y, Akhlaghi Farsi A, Sleep Parameters and the Factors Affecting the Quality of Sleep in Patients Attending Selected Clinics of Rasoul-e-Akram Hospital. Journal of Iran University of medical Sciences 2008; 15(58): 31-38.

31. Lakzai H, Shadadi H, Abdollahimohammad A, Dahmardeh M. Evaluation of serum ferritin levels in patients with thalassemia major in Imam-Ali hospital of Zabol in 2011. Journal of Zabol University of medical Sciences 2013; 5(1): 27-31.

32. Mojtahedzadeh, F; Thalassemia diseases; Research Deputy of Mazandaran Medical Sciences University, 2010; 197-198.

33. Behrman R.E, Kliegman R.M, Arvin A,M:
Nelson Textbook of pediatrics. 15 ed W.B Saunders co 1996; 1402. 2198.

34. Mollahoseini Sh, Mohammadzadeh Sh, Kamali P, Tavakoli Shoshtari M. Frequency of sleep disorder and restless legs syndrome in patients referred to hospitals affiliated to Tehran University of Medical Sciences in 1382. Journal of Medical Sciences, Islamic Azad University 2005; 15(1): 27-30.

35. Talaeepoor S. Compare serum ferritin level in thalassemia major patients in the group treated with deferoxamine alone and the group treated with deferoxamine and Deferasirox in the city of Bandar Abbas in 1391-1392. Bandar Abbas University of Medical Sciences 2014; thesis

36. Lamberg L. Insomnia shows strong link to psychiatric disorders. Psychiatric News 2005; 40: 21-3. 